\title{
Interaction of influenza virus NSI protein with growth arrest-specific protein 8
}

\author{
Lixia Zhao ${ }^{\dagger 1,2}$, Long Xu ${ }^{\dagger 1}$, Xiaowei Zhou ${ }^{1}$, Qingyu Zhu ${ }^{2}$, Zhixin Yang1, \\ Chuanfu Zhang ${ }^{1}$, Xudong Zhu ${ }^{1}$, Mengbin $\mathrm{Yu}^{1}$, Yingying Zhang1, \\ Xinghui Zhao ${ }^{2}$ and Peitang Huang*1
}

\begin{abstract}
Address: ${ }^{1}$ Laboratory of protein engineering, Beijing Institute of Biotechnology, Beijing 100071, PR China and ${ }^{2}$ State key laboratory of pathogen and biosecurity, Beijing Institute of Microbiology and Epidemiology, Beijing 100071, PR China

Email: Lixia Zhao - happylisa2008@yahoo.com.cn; Long Xu - xu_long@126.com; Xiaowei Zhou - amms832@126.com; Qingyu Zhu - qingyzh@yahoo.com.cn; Zhixin Yang - yy_xiao@126.com; Chuanfu Zhang - hnzcf@126.com; Xudong Zhu - xd_zhu@263.net; Mengbin Yu - xinwen23@163.com; Yingying Zhang - xray19@126.com; Xinghui Zhao - zhao4work@yahoo.com.cn; Peitang Huang* - hpt@hotmail.com

* Corresponding author †Equal contributors
\end{abstract}

Published: 9 December 2009

Virology Journal 2009, 6:218 doi:10.1186/1743-422X-6-218

This article is available from: http://www.virologyj.com/content/6/1/218

(c) 2009 Zhao et al; licensee BioMed Central Ltd.

This is an Open Access article distributed under the terms of the Creative Commons Attribution License (http://creativecommons.org/licenses/by/2.0), which permits unrestricted use, distribution, and reproduction in any medium, provided the original work is properly cited.
Received: 19 August 2009
Accepted: 9 December 2009

\begin{abstract}
NSI protein is the only non-structural protein encoded by the influenza $A$ virus, and it contributes significantly to disease pathogenesis by modulating many virus and host cell processes. A twohybrid screen for proteins that interact with NSI from influenza $A$ yielded growth arrest-specific protein 8 . Gas8 associated with NSI in vitro and in vivo. Deletion analysis revealed that the Nterminal 260 amino acids of Gas8 were able to interact with NSI, and neither the RNA-binding domain nor the effector domain of NSI was sufficient for the NSI interaction. We also found that actin, myosin, and drebrin interact with Gas8. NSI and $\beta$-actin proteins could be coimmunoprecipitated from extracts of transfected cells. Furthermore, actin and Gas8 co-localized at the plasma membrane. These results are discussed in relation to the possible functions of Gas 8 protein and their relevance in influenza virus release.
\end{abstract}

\section{Introduction}

Influenza A viral NS1 protein is a multifunctional protein that is capable of both protein-protein and protein-RNA interactions [1]. It binds non-specifically to doublestranded RNA and specifically to protein targets. NS1 binds directly to $\mathrm{p} 85 \beta$ to activate phosphatidylinositol 3 kinase signaling [2]. It binds a cleavage and polyadenylation specificity factor to inhibit the maturation and export of host antiviral mRNAs, and it inhibits poly(A)-binding protein II [3]. NS1 protein interacts with the viral RNA polymerase complex [4], the eukaryotic translation initiation factor eIF4GI [5], NS1-I [6], NS1-BP [7], Staufen [8], and nucleolin [9]. Association of NS1 protein with host factors may affect apoptosis $[10,11]$.

Growth arrest-specific genes are expressed preferentially in cultured cells that have entered a quiescent state following serum deprivation or growth to confluence. To date, eleven GAS genes have been identified that act in a variety of biological functions, including the control of microfilament organization [12], nerve cell growth or differentiation [13], apoptosis [14], tyrosine kinase receptor activity [15], and negative and positive control of the cell cycle $[16,17]$. No sequence similarity or common structural fea- 
tures have been found among the GAS genes or proteins [18].

GAS8, also known as GAS11, is located at $16 \mathrm{q} 24.3$ and was found to be a common deletion present in breast and prostate carcinomas. It was viewed as a potential tumor suppressor gene. The GAS8 gene consists of 11 exons spanning approximately $25 \mathrm{~kb}$. Northern blot analysis identified two ubiquitously expressed mRNAs of 3.4 and $1.8 \mathrm{~kb}$ in length. Another gene, C16orf3, lies within intron 2 of GAS8, and is transcribed in the opposite orientation of GAS8 [19].

Gas8 protein associates with microtubules in vitro and in vivo. Deletion analysis identified a microtubule-binding domain (GMAD) and a region that attenuates Gas8microtubule interactions (IMAD) [20]. Gas8 homologs in Trypanosoma brucei and Chlamydomonas reinhardtii are integral components of the flagellar axoneme that regulates flagellar beating. The GAS8 gene is also expressed in a variety of mammalian cells that lack motile cilia. In COS7 cells, Gas8 is localized to the Golgi apparatus. This localization is dependent on intact microtubules and is regulated by the cell cycle, as Gas8 is dispersed throughout the cytoplasm as cells progress through mitosis [21].

In adult mice, GAS 8 mRNA and protein are found predominantly in the testes, where expression is regulated during the post-meiotic development of male gametocytes [22].

We isolated Gas8 in a two-hybrid screen for proteins that interact with the influenza A virus protein NS1. We found that NS1 and Gas8 co-localize. Gas8 also interacted with actin, myosin, and drebrin.

\section{Materials and methods Cells and cell culture}

293FT, Hela, CV-1, NIH3T3, and BHK21 cells were maintained in Dulbecco's Modified Eagle's Medium (DMEM) containing $10 \%$ heat-inactivated fetal calf serum (HyClone). A549 cells were maintained in McCoy's 5A medium supplemented with 10\% heat-inactivated fetal calf serum.

\section{Construction of plasmids}

To generate the NS1 expression construct for the CytoTrap two-hybrid system, cDNAs encoding the NS1 proteins of A/Swine/Colorado/1/77 (H3N2) were amplified using the primers listed in Table 1 and cloned into pSos, creating pSos-NS1. To generate an $\mathrm{N}$-terminally myc-tagged NS1 expression construct, the open reading frames encoding NS1 were amplified using the primers listed in Table 1. The PCR products were cloned into pCMV-Myc via the SalI and NotI restriction sites to create pCMV-Myc-NS1. The expression plasmids pGEX-6P-1-NS1 and pEGFP-N3NS1 were generated by inserting an NS1 cDNA corresponding to amino acids 1 to 237 between the EcoRI/NotI sites of pGEX-6P-1 and the EcoRI/KpnI sites of pEGFP-N3 (NEB), respectively. The GAS8 gene purchased from Proteintech was cloned by PCR using a primer pair specific for the human cDNA (GeneBank accession no. NM 001481). The products were cloned into pDsRedExpress-C1 and pCMV-Tag 2B respectively, creating pDsRed-GAS8 and FLAG-tagged GAS8. Different NS1 and Gas8 deletion mutants were generated by PCR, as above.

Table I: Primers used in this study

\begin{tabular}{|c|c|c|}
\hline \multirow[t]{2}{*}{ Purpose } & \multicolumn{2}{|c|}{ Primer sequence (5'-3') } \\
\hline & Forward & Reverse \\
\hline $\begin{array}{l}\text { NSI } \\
\text { pSos }\end{array}$ & CGGTCGACGATGGATTCCAACACTGTGT & ACGTGCGGCCGC ATCAGCCATCTTATCTCTTC \\
\hline $\begin{array}{c}\text { NSI } \\
\text { pCMV-Myc }\end{array}$ & ACGCGTCGACCATGGATTCCAACACTGTGTC & TTGCGGCCGC TCAATCAGCCATCTTATCTC \\
\hline $\begin{array}{c}\text { NSI } \\
\text { pEGFP-N3 }\end{array}$ & CCGAATTCTATGGATTCCAACACTGTGTC & GGGGTACC ATCAGCCATCTTATCTCTTC \\
\hline $\begin{array}{c}\text { NSI } \\
\text { pGEX-6P-I }\end{array}$ & CCGAATTCATGGATTCCAACACTGTGTC & TTGCGGCCGC TCAATCAGCCATCTTATCTC \\
\hline $\begin{array}{c}\mathrm{NSI}_{1-80} \\
\text { pCMV-Myc }\end{array}$ & ACGCGTCGACC ATGGATTCCAACACTGTGTC & TTGCGGCCGC TCAGGTCATTGTAAGCGCCTC \\
\hline $\begin{array}{c}\mathrm{NSI}_{81-238} \\
\text { pCMV-Myc }\end{array}$ & ACGCGTCGACC ATGGCCTCCACACCTGC & TTGCGGCCGC TCAATCAGCCATCTTATCTC \\
\hline GAS8 pDsRed-Express-CI & GGAATTCGATGGCACCGAAAAAGAAAGGGAAGA & GGGGTACCCGTCGGGGTGCCCACCAGTCCCGC \\
\hline $\begin{array}{c}\text { GAS8 } \\
\text { pCMV-Tag 2B }\end{array}$ & CGGAATTCATGGCACCGAAAAAGAAAGGGAAGA & CCCTCGAGTTACGTCGGGGTGCCCACCAGTCC \\
\hline $\begin{array}{c}\text { GAS8 }_{1-260} \\
\text { pCMV-Tag 2B }\end{array}$ & CGGAATTCATGGCACCGAAAAAGAAAGGGAAGA & CCCTCGAGTTACTTCCGCATGTCCTCCATCTG \\
\hline $\begin{array}{c}\text { GAS8 }_{260-478} \\
\text { pCMV-Tag 2B }\end{array}$ & CGGAATTCAAGGAGGACCACCTGGAGAGGG & CCCTCGAGTTACGTCGGGGTGCCCACCAGTCCCGC \\
\hline
\end{tabular}


All primers are listed in Table 1 and all constructs were confirmed by sequence analysis.

\section{Isolation of NSI-interacting CDNA clones using the yeast interaction trap}

The CytoTrap two-hybrid system (Stratagene) was used to identify and isolate CytoTrap XR premade library (Merck) cDNAs encoding NS1 binding factors, according to the manufacturer's instructions. In brief, the $\mathrm{CDC} 25 \mathrm{H}(\alpha)$ yeast strain was transformed with the bait plasmid pSosNS1 and the library DNA purified from the premade libraries, in which human lung cDNAs were conditionally expressed as fusions with a myristoylation membrane localization signal from a GAL1 promoter. A total of $1.09 \times 10^{7}$ primary transformants were screened for an interaction, as determined by their ability to grow on minimal synthetic medium in the absence of leucine and uracil in plates containing glucose but not galactose at $23^{\circ} \mathrm{C}$. Putative clones were isolated by their ability to grow on minimal synthetic medium in the absence of leucine and uracil in plates containing galactose but not glucose at $37^{\circ} \mathrm{C}$. The interaction was verified by retransformation of pSos-NS1 with pMyr cDNA plasmid DNA isolated from the putative clones previously identified.

\section{Transient transfection and subcellular localization}

The NS1 gene from avian influenza virus A/Swine/Colorado/1/77 (H3N2) was kindly provided by ShuZhang Feng. 293FT cells were transfected with pDsRed-GAS8 and pDsRed-GAS8/pEGFP-NS1 using Lipofectamine 2000 (Invitrogen). After twenty-four hours, cells were fixed with 4\% paraformaldehyde and washed with PBS. Nuclei were stained with DAPI (Sigma). The subcellular localization of the pDsRed-GAS8 and pEGFP-NS1 fusion proteins was detected using a Zeiss LSM 510 META microscope equipped with a $100 \times$ objective lens (Zeiss).

\section{GST pulldown assays}

GST and GST-NS1 proteins were expressed in Escherichia coli BL21 induced with isopropyl- $\beta$-D-thiogalactopyranoside (IPTG) (Merck). Five milligrams of GST and GST-NS1 were adsorbed from bacterial lysates to MagneGST ${ }^{\mathrm{TM}}$ particles, as recommended by the manufacturer (Promega). The immobilized proteins were mixed with $2 \mathrm{mg}$ of 293FT cell extracts containing myc-tagged Gas 8 protein in binding buffer ( $50 \mathrm{mM}$ Tris-HCl, $150 \mathrm{mM} \mathrm{NaCl}, 1.0 \%$ Triton X$100, \mathrm{pH} 7.6)$ containing a protease inhibitor mix (Roche) and incubated for $1 \mathrm{~h}$ at room temperature on a rotating platform. Beads were precipitated and washed three times with $500 \mu \mathrm{l}$ of binding buffer. Proteins bound to GST and GST-NS1 were analyzed by SDS-PAGE, followed by western blot analysis with anti-myc antibody (Invitrogen).

\section{Immunoprecipitation and immunoblotting}

293FT Cells were transfected with pCMV-Tag 2B-Gas8 and pCMV-Myc-NS1 expression plasmids. Following transfection for $48 \mathrm{~h}$, the cells were lysed in RIPA buffer $(150 \mathrm{mM}$ $\mathrm{NaCl}, 1 \% \mathrm{NP}-40,0.5 \%$ deoxycholic acid, 0.1\% SDS, 50 $\mathrm{mM}$ Tris- $\mathrm{HCl}, \mathrm{pH} 7.5)$ containing protease inhibitors. Lysates were immunoprecipitated (IP) using anti-FLAG M2 (or anti-Myc) mouse monoclonal antibody (Sigma). Immunoprecipitated proteins were immunoblotted (IB) with anti-myc (or anti-FLAG) antibody. Positive bands were detected with the ECL western blotting detection reagent (Amersham), and the image was visualized with CL$\mathrm{X}$ posure ${ }^{\mathrm{TM}}$ film (Pierce). To identify the immunoprecipitated Gas8-interacting proteins, 293FT cells were transfected with pCMV-Tag 2B-Gas8 or pCMV-Tag $2 \mathrm{~B}$. Following transfection for $48 \mathrm{~h}$, the cells were lysed in RIPA buffer. Lysates were IPed using anti-FLAG M2 mouse monoclonal antibody, and the immunoprecipitated proteins were resolved by SDS-PAGE and Coomassie stained. The peptide mass fingerprint analysis was performed by the National Center for Biomedical Analysis, Beijing.

\section{Immunofluorescence and confocal microscopy}

CV-1 cells were transfected with pDsRed-GAS8. After 24 hours, the cells were washed with $1 \mathrm{ml}$ of PBS and fixed in methanol:acetone $(\mathrm{v} / \mathrm{v}=1: 1)$. After PBS washes, rat anti$\beta$-actin serum (Sigma) was applied at a dilution of 1:200, followed by a one-hour incubation at room temperature. The cells were washed with PBS and incubated with fluorescein-conjugated affinipure goat anti-mouse IgG (Zhongshan Goldenbridge Biotechnology Co., China) at a dilution of 1:50. Cells were washed with PBS and examined with a confocal microscope.

\section{Results \\ Isolation of NSI binding proteins}

The CytoTrap system makes use of the yeast $S$. cerevisiae temperature-sensitive mutant strain cdc25H. The strain grows normally at $23^{\circ} \mathrm{C}$, but does not grow at $37^{\circ} \mathrm{C}$. The CytoTrap system is based on the ability of the human Sos protein, which is the human homologue of the yeast CDC25 gene, to complement the cdc25 defect and to activate the yeast Ras signaling pathway. Expression of hSos and its subsequent localization to the plasma membrane allows the $\mathrm{cdc} 25 \mathrm{H}$ yeast strain to grow at $37^{\circ} \mathrm{C}$. The localization of Sos to the plasma membrane occurs through the interaction of two-hybrid proteins. We used a pSosNS1 fusion protein to screen a human lung cDNA plasmid library, in which cDNA-encoded proteins were conditionally expressed as translational fusions with a pMyr. Expression of the pMyr fusion proteins is induced in the presence of galactose and repressed by glucose. Twentyfour library plasmids were isolated from $1.09 \times 10^{7}$ transformants based on their ability to grow on minimal syn- 
thetic medium in the absence of leucine and uracil in plates containing galactose but not glucose at $37^{\circ} \mathrm{C}$. Further identification of putative positive plasmids was performed by co-transformation with pSos-NS1, and 15 out of 24 were confirmed as positive plasmids. After DNA sequencing, 8 clones of different gene were obtained. Here, we report the analysis of one of the human cDNAs isolated from these library plasmids that encodes growth arrest-specific gene 8 .

\section{NSI interacts with Gas8 in vitro and in vivo}

To confirm the interaction between NS1 and Gas8 suggested by the two-hybrid results, two experiments were performed. First, GST pull-down assays we performed to find proteins that bind to a GST-NS1 protein in 293FT cell lysates expressing myc-tagged Gas8. Gas8 was efficiently precipitated by a GST-NS1 fusion protein but not by GST (Fig. 1A), indicating that NS1 interacts with Gas8 in vitro. Next, the interaction of NS1 and Gas8 in vivo was investigated by co-immunoprecipitation (co-IP). When lysates of 293FT cells expressing FLAG-tagged Gas8 were immunoprecipitated with anti-FLAG antibodies, myc-tagged
NS1 protein was detected in the precipitate. Similarly, Gas8 protein could also be precipitated by NS1 (Fig. 1B).

\section{Protein-protein interaction domains}

To identify the region of NS1 protein involved in the interaction with Gas 8 protein, in vivo binding experiments were carried out. 293FT cells were transfected with the plasmids pCMV-Tag 2B-Gas8 and pCMV-myc-NS $1_{1-238}$ or one of its truncated derivatives: pCMV-myc-NS1 $1_{1-80}$, pCMV-myc-NS1 $1_{81-238}$, or pCMV-myc. When lysates of 293FT cells transfected with the different plasmids mentioned above were immunoprecipitated with anti-myc antibodies, no FLAG-tagged Gas8 protein was detected in the cells expressing truncated NS1 (Fig. 2A). The proteinprotein interaction domain of Gas8 was identified by IP assays, as described above, this time using Gas 8 deletion mutants. Gas $8_{1-260}$ interacted with NS1. In contrast, no interaction with Gas $8_{260-478}$ was observed (Fig. 2B).

\section{Gas8 and NSI proteins co-localize}

Based on the two-hybrid and in vitro binding results, we hypothesized that Gas8 and NS1 interact in vivo. If this

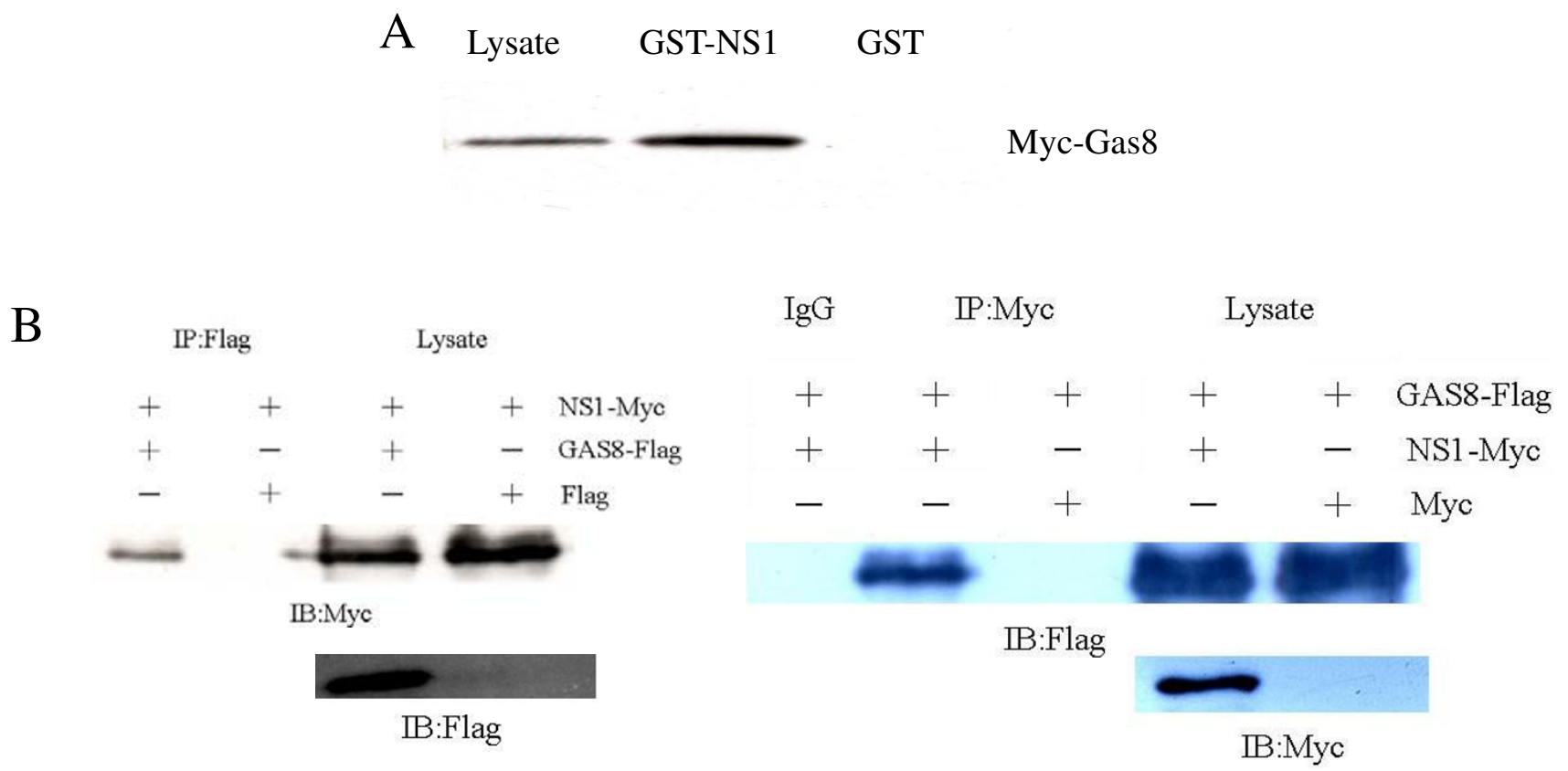

\section{Figure I}

In vitro and in vivo interaction between NSI and Gas8. A GST pull-down analysis. Lysates from cells expressing mycGas8 were mixed with GST or GST-NSI, and bound proteins were analyzed by anti-myc western blot. Left lane, cell lysate; middle and right lanes, proteins bound to GST-NSI and GST, respectively. Gas8 was precipitated by a GST-NSI fusion protein but not by GST. (B) Western blot of NSI and Gas8 co-IPs. Left: Cells were co-transfected with a plasmid expressing myctagged NSI and either a plasmid expressing FLAG-tagged Gas8 or the empty vector. Cell lysates were IPed with anti-FLAG antibody (left two lanes) or run on a gel without immunoprecipitation (right two lanes). Samples were probed with anti-myc and anti-FLAG antibody. Right: IP with anti-myc antibody and IB with anti-FLAG and anti-myc antibody. The results showed that NSI could be precipitated with Gas8 and Gas8 could also be precipitated with NSI. 
A

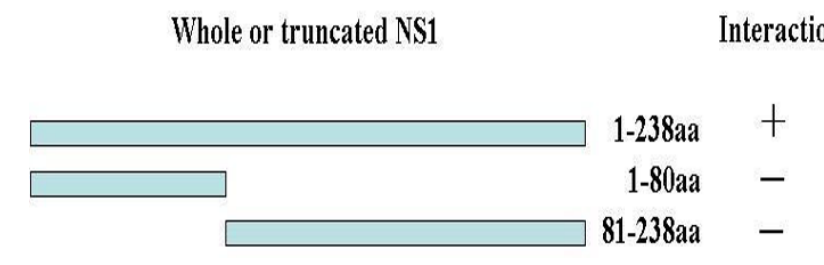

B

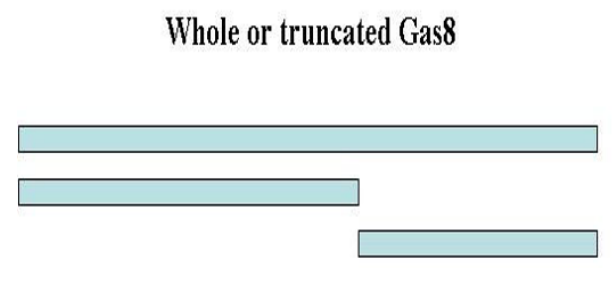

Interaction

$\begin{aligned} 1-478 a a & + \\ 1-260 a a & + \\ 260-478 a a & -\end{aligned}$

Lysate IP:Myc

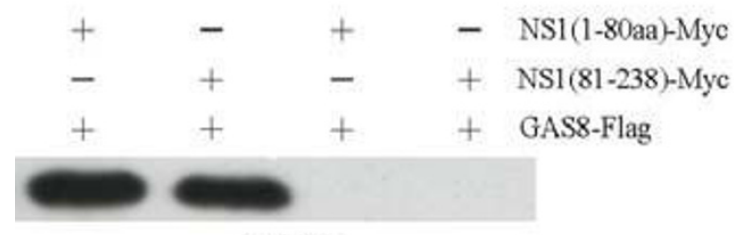

IB.Flag

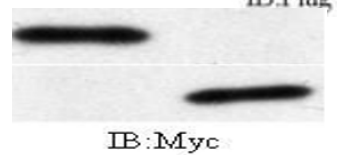

Lysate IPFlag

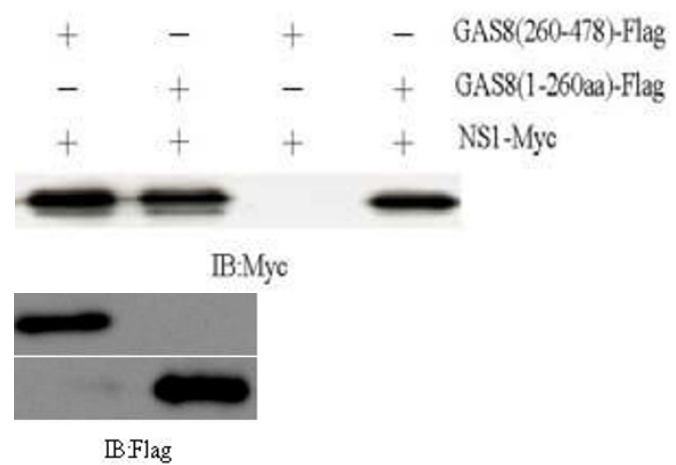

Figure 2

Protein-protein interaction domains. (A) Mapping of the NSI domain interacting with Gas8. 293FT cells were transfected with pCMV-Tag 2B-Gas8 and one of its truncated derivatives: pCMV-myc-NSI ${ }_{1-80}$, pCMV-myc-NSI 8 I-238 $_{2}$, or pCMV-myc. After incubation for $36 \mathrm{~h}$, cell lysates were IPed with anti-myc antibody and probed with anti-FLAG and anti-myc antibody. (B) Schematic representation of Gas8 proteins. Polypeptides corresponding to Gas $8_{\mid-478}$, Gas $8_{\mid-260}$, and Gas $8_{260-478}$ are shown. The corresponding amino acids are indicated on the right. 293FT cells were transfected with pCMV-myc-NSI and one of its truncated derivatives: PCMV-Tag 2B-Gas8 ${ }_{\mid-260}$, PCMV-Tag 2B-Gas8 ${ }_{260-478}$, or PCMV-Tag 2B. Cell lysates were IPed with anti-myc antibody and probed with anti-FLAG and anti-myc antibody. +: protein-protein interaction; -: no protein-protein interaction.

interaction is physiologically relevant, we would expect the two proteins to occupy the same intracellular compartment. To test this prediction, a Gas8-DsRed and an NS1-GFP fusion were co-expressed in 293FT cells. The Gas8 protein has been reported to localize to the Golgi apparatus [21]. The NS1 protein has been found in large foci in the nucleus $[23,24]$, a localization that was confirmed in 293FT cells (Fig. 3). When both proteins were co-expressed, fluorescently tagged Gas8 and NS1 exhibited juxtanuclear co-localization. These results suggest that Gas8 can modify the location of NS1.

\section{Gas8 interacts with actin, myosin, and drebrin}

To find cellular proteins that interact with Gas8, coimmunoprecipitated proteins were identified by peptide mass spectrometry (Fig. 4A). Peptide fingerprinting iden- tified the proteins myosin-9, drebrin E2, and actin. The actin-binding protein drebin has been localized to the apical plasma membrane together with a pool of submembranous F-actin, which is hypothesized to modulate actin-myosin interactions in dendritic spines $[25,26]$. Myosin 9 may function in intracellular vesicle transport [27]. CV-1 cells transfected with pDsRed-GAS8 were analyzed by confocal microscopy and immunofluorescence using anti- $\beta$-actin antibody. The results showed that Gas 8 and $\beta$-actin co-localize at the plasma membrane, but not in the cytoplasm (Fig. 4B). Their association was confirmed by co-IP (Fig. 4C).

\section{Discussion}

Gas8 was obtained from a two-hybrid screen for cytoplasmic interactions with the influenza A protein NS1. The 


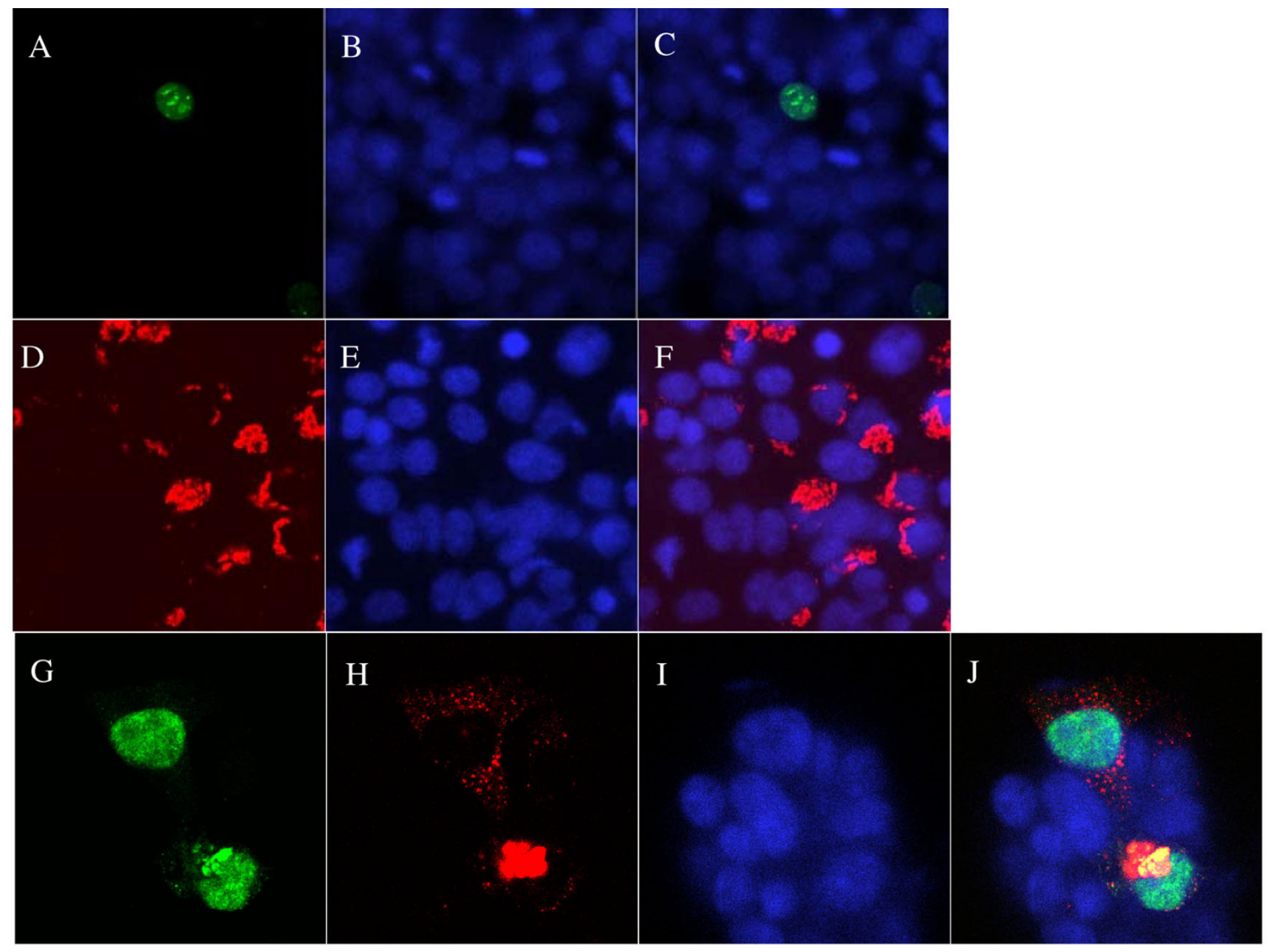

\section{Figure 3}

Confocal micrographs of NSI and Gas8 in 293FT cells. Panels (A) and (G): GFP. Panels (D) and (H): DsRed. Panels (B), (E), (I): DAPI. Panels (C), (F), (J): merged images. Top row: cells transfected with pEGFP-NSI showing nuclear localization. Middle row: cells transfected with pDsRed-GAS8 showing juxtanuclear localization. Bottom row: co-expression of NSI and Gas8 showing juxtanuclear localization.

interaction was confirmed in vitro and in vivo with GSTpulldown and co-IP assays. Identification of the NS1 domain that interacts with Gas8 revealed that neither the RNA binding domain nor the effector domain of NS1 alone could interact with Gas8, in contrast to a previously reported NS1-interacting protein[28]. Deletion analysis revealed that the N-terminal 260 amino acids of Gas 8 were able to interact with NS1. This domain corresponds to IMAD and GMAD domain.

Examination of the localization of Gas 8 protein in cells revealed that two types of localization exist: Golgi and cytoplasmic. This phenomenon was even observed in the same types of cells. The Golgi apparatus localization is dependent on intact microtubules and is cell-cycle regulated, as Gas8 is dispersed throughout the cytoplasm as cells progress through mitosis[21].
Since the function of Gas8 has not yet been determined, the effect of the association between Gas 8 and NS1 on virus infection is unknown. The mammalian Gas 8 gene is a possible tumor suppressor that was previously identified as one of several genes that are up-regulated upon growth arrest[19]. Cell proliferation was not inhibited when CV1 and DU145 cells were transfected with the recombinant plasmid pCMV-Tag 2B-GAS8. Gas8 was expressed in HeLa, CV-1, A549, and DU145 cells. NS1 inhibited the maturation of GAS8 mRNA. This is consistent with the fact that transient expression of NS1 in mammalian cells leads to retention of poly(A) RNA in the nucleus and inhibition of pre-mRNA splicing[1,29]. In order to study the function of Gas8, we attempted to abrogate the expression of Gas8. Unfortunately, an effective target for RNAi was not found. 

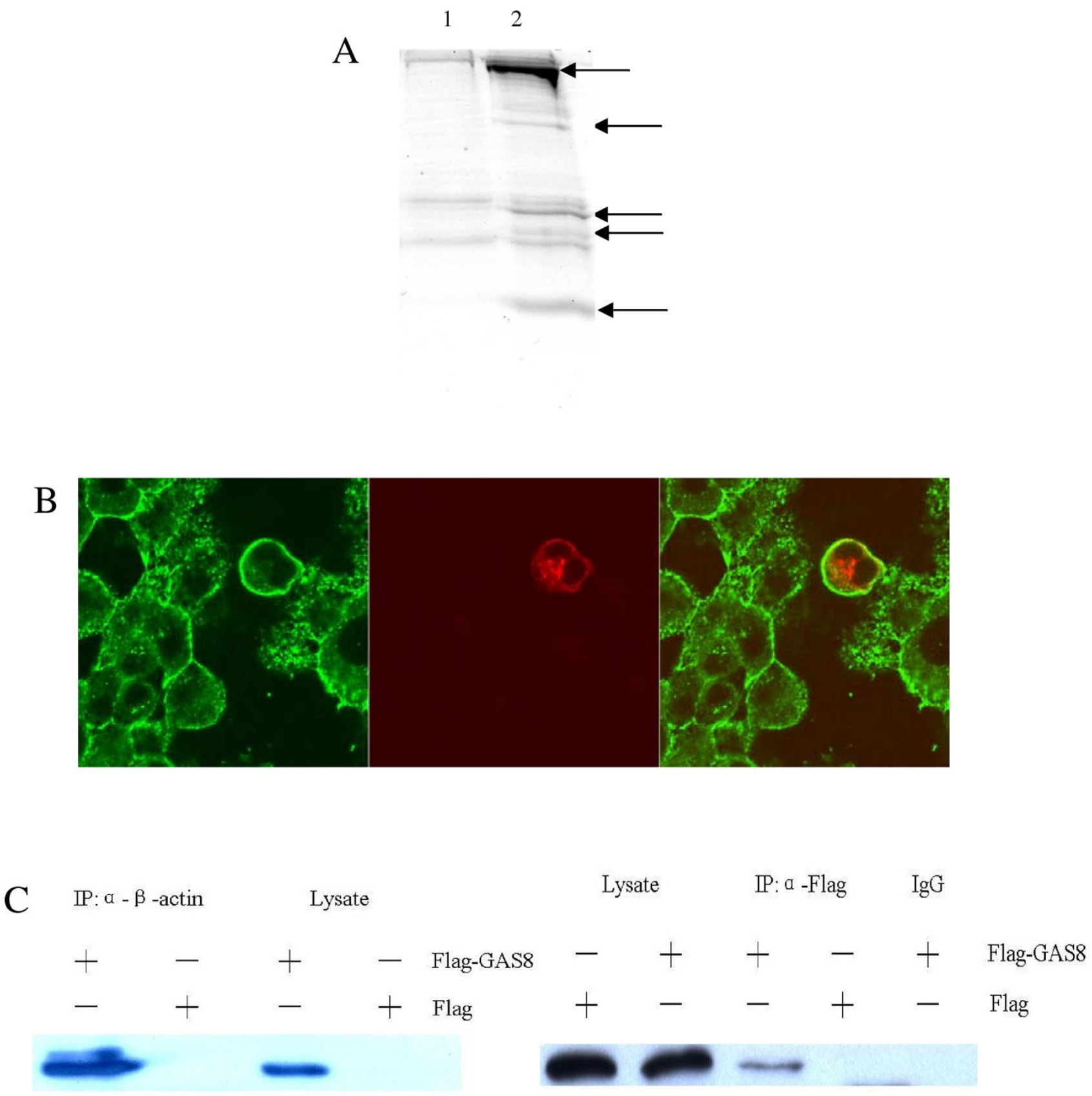

IB: a -Flag

IB: $\alpha-\beta$-actin

\section{Figure 4}

Interaction between $\mathbf{G a s} 8$ and $\beta$-actin. (A)SDS-PAGE analysis of proteins that co-IPed with Gas8. 293FT cells were transfected with PCMV-Tag 2B-GAS8 or PCMV-Tag 2B, and cell extracts were immunoprecipitated with anti-FLAG antibody. The gels were Coomassie stained. I: pCMV-Tag 2B/293FT, 2: pCMV-Tag 2B-GAS8/293FT. Arrows indicate proteins that co-IPed with Gas8. (B) Gas8 co-localizes with $\beta$-actin at the plasma membrane. CV-I cells were transfected with pDsRed-GAS8. After $24 \mathrm{~h}$, the cells were fixed and probed with an antibody against $\beta$-actin. The merged image shows co-localization of Gas 8 (red) and $\beta$-actin (green) at the plasma membrane. (C) Interaction of Gas8 and $\beta$-actin proteins in vitro. 293FT cells were transfected with PCMV-Tag 2B-GAS8 (FLAG-Gas8) or PCMV-Tag 2B (FLAG). Soluble cell lysates were IPed with anti-FLAG or anti- $\beta$-actin antibody and probed with anti-FLAG (left) or anti- $\beta$-actin antibody (right). 
We identified proteins that interact with Gas8 by immunoprecipitation and found that Gas8 co-localizes with actin at the plasma membrane, but not in the cytoplasm. We speculate that this may be the result of its association with drebrin E2, which is the only protein of the myosin9-drebrin E2-actin complex reported to be localized to the apical plasma membrane with actin[26]. However, successful expression of the drebrin E2 protein was not achieved, and therefore a direct interaction between Gas8 and drebrin still needs to be confirmed. Drebrin has been reported to modulate actin-myosin interactions in dendritic spines, and myosin 9 may function in intracellular vesicle transport $[26,27]$. Therefore we examined the effect of overexpression of Gas8 on the maturation of progeny virions and found that Gas 8 could arrest the production of H3N2 progeny virions from cells.

Gas8 has been reported to interact with Rab3B. Rab3 subfamily proteins are enriched in neuronal and secretory cells, where they control regulated exocytosis through the interaction with the Rab3 effector proteins Rabphilin-3, $\operatorname{Rim} 1 / 2$, and Noc2 [30]. In fibroblasts and epithelial cells, Rab3B is involved in the transport of low-density lipoprotein receptors, vesicular stomatitis virus glycoprotein, and polymeric immunoglobulin receptors [31-33]. Overexpression of Noc2 inhibited the cell-surface transport of basolateral vesicular stomatitis virus glycoprotein. Consistent with the functions of these Gas8-associated proteins and the binding detected between Gas 8 and drebrin, actin, and myosin, we speculate that Gas 8 may affect the release of H3N2 progeny virions.

\section{Competing interests}

The authors declare that they have no competing interests.

\section{Authors' contributions}

LXZ and LX performed analysis of interaction between Gas8 and NS1 and wrote the manuscript. XWZ, QYZ, XDZ and PTH conceived the studies and participated in experimental design and coordination. CFZ isolated Gas8 from the library. ZXY and XHZ carried out viral replication in cell culture. MBY and YYZ participated in abrogation the expression of Gas8. All authors read and approved the final manuscript.

\section{Acknowledgements}

This study was supported by a grant from the National Key Technology R\&D Program of China (No.2006BAD06A0I).

\section{References}

I. Qiu Y, Krug RM: The influenza virus NSI protein is a poly(A)binding protein that inhibits nuclear export of mRNAs containing poly(A). J Virol 1994, 68:2425-2432.

2. Hale BG, Jackson D, Chen YH, Lamb RA, Randall RE: Influenza A virus NSI protein binds $\mathbf{p} 85$ beta and activates phosphatidylinositol-3-kinase signaling. Proc Natl Acad Sci 2006, 103:14194-14199.
3. Krug RM, Yuan WM, Noah DL, Anita Ghate Latham : Intracellular warfare between human influenza viruses and human cells: the roles of the viral NSI protein. Virol 2003, 309:18I-189.

4. Marion RM, Zurcher T, Luna S, Ortin J: Influenza virus NSI protein interacts with viral transcription-replication complexes in vivo. J Gen Virol 1997, 78:2447-245I.

5. Burgui I, Aragon T, Ortin J, Nieto A: PABPI and elF4GI associate with influenza virus NSI protein in viral mRNA translation initiation complexes. J Gen Virol 2003, 84:3263-3274.

6. Wolff $\mathrm{T}, \mathrm{O}$ 'Neill R, Palese P: Interaction cloning of NSI-I, a human protein that binds to the nonstructural NSI proteins of influenza A and B viruses. I Virol 1996, 70:5363-5372.

7. Wolff T, O'Neill R, Palese P: NSI-binding protein (NSI-BP): a novel human protein that interacts with the influenza $A$ virus nonstructural NSI protein is relocalized in the nuclei of infected cells. J Virol 1998, 72:7170-7|80.

8. María Falcón A, Fortes P, María Marión R, Beloso A, Ortin J: Interaction of influenza virus NSI protein and the human homologue of Staufen in vivo and in vitro. Nucleic Acids Res 1999, 27:224I-2247.

9. Murayama R, Harada Y, Shibata T, Kuroda K, Hayakawa S, Shimizu K, Tanaka T: Influenza A virus non-structural protein I (NSI) interacts with cellular multifunctional protein nucleolin during infection. Biochem Bioph Res Co 2007, 362:880-885.

10. Cherry SS, Sissoko ND, Neumann G, Kawaoka Y, Hinshaw VS: Influenza virus NSI protein induces apoptosis in cultured cells. J Virol 200I, 75:7875-788I

II. Zhirnov OP, Konakova TE, Wolff T, Klenk HD: NSI protein of influenza Avirus down-regulates apoptosis. J Virol 2002, 76:1617-1625.

12. Brancolini C, Bottega S, Schneider C: Gas2, a growth arrest-specific protein, is a component of the microfilament network system. J Cell Biol 1992, I I 7:125I-I26I.

13. Suter UA, Snipes G]: Peripheral myelin protein 22: Facts and hypotheses. J Neurol Res 1995, 40:145-151.

14. Lee KK, Leung AK, Tang MK, Cai DQ, Schneider C, Brancolini C, Chow PH: Functions of the growth arrest specific I gene in the development of the mouse embryo. Dev Biol 200I, 234: 188-203.

15. Hafizi S, Dahlback B: Gas6 and protein S Vitamin K-dependent ligands for the Axl receptor tyrosine kinase Subfamily. FEBS J 2006, 273:523।-5244.

16. Zoidl G, Blass-Kampmann S, D'Urso D, Schmalenbach C, Muller HW: Retroviral-mediated gene transfer of the peripheral myelin protein PMP22 in Schwann cells modulation of cell growth. EMBO J 1995, I 4: I I 22- I I28.

17. Liu Y, May NR, Fan CM: Growth Arrest Specific Gene I is a positive growth regulator for the cerebellum. Dev Biol 200I, 236:30-45.

18. Ju YT, Chang AC, She BR, Tsaur ML, Hwangi HM, Chao CC, Cohen $\mathrm{SN}$, Sue LC: gas7 : A gene expressed preferentially in growtharrested fibroblasts and terminally differentiated Purkinje neurons affects neurite formation. Proc Natl Acad Sci 1998, 95: I |423-I | 428

19. Whitmore SA, Settasatian C, Crawford J, Lower KM, Mccallum B, Seshadri R, Cornelisse CJ, Moerland EW, Cleton-Jansen AM, Tipping AJ, Mathew CG, Savnio M, Savoia A, Verlander P, Auerbach AD, Carola VB, Pronk JC, Doggett NA, Callen DF: Characterization and screening for mutations of the growth arrest-specific II (GASII) and Cl6orf3 genes at $16 \mathrm{q} 24.3$ in breast cancer. Genomics 1998, 52:325-331.

20. Bekker JM, Colantonio JR, Stephens AD, Thomas Clarke W, King SJ, Hill KL, Crosbie RH: Direct interaction of Gas I I with microtubules: implications for the dynein regulatory complex. Cell Motility and the Cytoskeleton 2007, 64:46I-473.

21. Colantonio JR, Bekker JM, Kim SJ, Morrissey KM, Crosbie RH, Hill KL: Expanding the role of the dynein regulatory complex to nonaxonemal functions: association of GASII with the Golgi apparatus. Traffic 2006, 7:538-548.

22. Yeh SD, Chen YJ, Chang AY, Ray R, She BR, Lee WS, Chiang HS, Cohen SN, Sue LC: Isolation and properities of Gas8, a growth arrest-specific gene regulated during male gametogenesis to produce a protein associated with the sperm motility apparatus. J Biol Chem 2002, 27:63II-63I7. 
23. Greenspan $D$, Palese $P$, Krystal M: Two nuclear location signals in the influenza virus NSI nonstructural protein. J Virol 1988, 62:3020-3026.

24. Mele'n K, Kinnunen L, Fagerlund R, Ikonen N, Twu KY, Krug RM, Julkunen I: Nuclear and nucleolar targeting of Influenza $A$ virus NSI protein:striking differences between different virus subtypes. J Virol 2007, 8I:5995-6006.

25. Hayashi K, Ishikawa R, Ye LH, He XL, Takata K, Kohama K, Shirao T: Modulatory role of drebrin on the cytoskeleton within dendritic spines in the rat cerebral cortex. J Neurosci 1996, 16:7|6I-7I70.

26. Keon BH, Jedrzejewski PT, Paul DL, Goodenough DA: Isoform specific expression of the neuronal F-actin binding protein, drebrin, in specialized cells of stomach and kidney epithelia. J Cell Sci 2000, I I 3:325-336.

27. Linz-McGillem LA, Alliegro MC: Myosin II in retinal pigmented epithelial cells: evidence for an association with membranous vesicles. Exp Eye Res 2003, 76:543-552.

28. Benjamin GH, Richard ER, Juan O, David J: The multifunctional NSI protein of influenza A viruses. J Gen Virol 2008, 89:2359-2376

29. Fortes P, Beloso A, Ortín J: Influenza virus NSI protein inhibits pre-mRNA splicing and blocks mRNA nucleocytoplasmic transport. EMBO J 1994, I 3:704-7II2.

30. Noriyuki N, Kunihiko A, Wakako S, Yumiko N, Kaho N, Hironori H, Takuya S: Interaction of Rab3B with microtubule-binding protein Gas8 in NIH 3T3 cells. Arch Biochem Biophys 2008, 474: I36-I 42.

31. Sidhu RS, Bhullar RP: Rab3B in human platelet is membrane bound and interacts with $\mathrm{Ca} 2 \mathrm{I} / \mathrm{Calmodulin}$. Biochem Bioph Res Co 200I, 289: 1039-1043.

32. Manabe S, Nishimura N, Yamamoto $Y$, Kitamura H, Morimoto S, Imai $M$, Nagahiro S, Seino S, Sasaki T: Identification and characterization of Noc2 as a potential Rab3B effector protein in epithelial cells. Biochem Bioph Res Co 2004, 3 16:21 8-225.

33. Weber E, jilling T, Kirk KL: Distinct functional properties of Rab3A and Rab3B in PCI 2 neuroendocrine cells. J Biol Chem 1996, 271:6963-697|.

Publish with Biomed Central and every scientist can read your work free of charge

"BioMed Central will be the most significant development for disseminating the results of biomedical research in our lifetime. "

Sir Paul Nurse, Cancer Research UK

Your research papers will be:

- available free of charge to the entire biomedical community

- peer reviewed and published immediately upon acceptance

- cited in PubMed and archived on PubMed Central

- yours - you keep the copyright 